\title{
Detección de espectro para radio cognitiva
}

\author{
Spectrum sensing for cognitive radio \\ Luis Fernando Pedraza ${ }^{1} \quad$ Felipe Forero $^{1} \quad$ Ingrid Patricia Páez ${ }^{2}$ \\ Recibido 28 de marzo de 2012, aceptado 11 de junio de 2012 \\ Received: March 28, $2012 \quad$ Accepted: June 11, 2012
}

\begin{abstract}
RESUMEN
Este artículo centra su atención en la capa física de las redes de radio cognitiva (CR), es decir, en las técnicas que se utilizan para detectar la presencia o ausencia de usuarios licenciados en las diferentes bandas del espectro electromagnético. Las técnicas presentadas, con sus ventajas y desventajas, están organizadas jerárquicamente como: técnicas fundamentales (detección de energía, filtro adaptado y características ciclo-estacionarias), técnicas basadas en las fundamentales o evolución (detección de forma de onda, identificación del radiotransmisor, detección de la potencia de fuga en el receptor) y técnicas alternativas típicas del análisis de imágenes (transformada Hough y Wavelet). Adicionalmente se describen los retos principales en la detección de usuarios licenciados junto con las diferentes dimensiones en las que se pueden hallar oportunidades de uso espectral eficiente por parte de los dispositivos de radio cognitiva.
\end{abstract}

Palabras clave: Radio cognitiva, detección de espectro, usuarios primarios, usuarios secundarios, asignación dinámica de espectro, huecos espectrales.

\begin{abstract}
This survey article focuses on the physical layer for cognitive radio $(C R)$ networks, namely the techniques to detect the presencelabsence of licensed users in the different spectrum bands. The techniques presented in this article, including their advantages and disadvantages, are organized in a hierarchical fashion as follows: fundamental detection techniques (energy-based detection, matched filter, and cyclostationarity features), fundamental-detection-based techniques (waveform detection, radio identification, localoscillator leakage power), and alternative techniques that are typical of the image analysis domain (Hough and Wavelet transforms). Additionally, the main challenges associated to licensed user detection are described together with the various spectral dimensions where cognitive radio devices can find new spectrum opportunities.
\end{abstract}

Keywords: Cognitive radio, spectrum sensing, primary users, secondary users, dynamic spectrum allocation, spectrum holes.

\section{INTRODUCCIÓN}

En la actualidad, las redes inalámbricas, tales como la red de telefonía celular o las redes tipo WiFi, comunican a casi todos los habitantes del planeta, brindando servicios como llamadas telefónicas convencionales, acceso a Internet, geoposicionamiento, etc. La principal ventaja de este tipo de redes es su movilidad, que las hace muy atractivas para los usuarios que necesitan disponer de una red portátil; sin embargo, esta fortaleza fundamental de las conexiones inalámbricas se ve opacada por la reducción en ancho de banda cuando este se compara con la capacidad de transmisión

1 Facultad de Tecnología. Universidad Distrital "Francisco José de Caldas”. Cr 7 N 40-53. Bogotá, Colombia. E-mail: 1fpedrazam@udistrital.edu.co; fforeror@udistrital.edu.co

2 Facultad de Ingeniería. Universidad Nacional de Colombia. Carrera 45 № 26-85. Bogotá, Colombia. E-mail: ippaezp@unal.edu.co 
de las redes cableadas. Para aumentar el ancho de banda de las redes inalámbricas se requiere cambiar el paradigma de asignación estática del espectro electromagnético. Se sugiere que gran parte del espectro asignado bajo las políticas actuales se usa de forma esporádica, lo que indica que el espectro está siendo subutilizado en gran medida [1]. En este escenario, los conceptos de CR son vistos como una solución que permitirá a los usuarios móviles grandes anchos de banda usando arquitecturas inalámbricas heterogéneas. Por supuesto, esta interacción entre arquitecturas impone retos importantes, los cuales van desde la estimación de las bandas de frecuencia que están en estado ocioso hasta el desarrollo de protocolos de enrutamiento propios de una asignación dinámica de recursos de red.

El marco de trabajo sugerido para estructurar el estudio de las redes de CR [2] establece la funcionalidad que deben ofrecer estos sistemas y propone tres aspectos fundamentales de diseño relacionados con la prevención de interferencia, el sentido permanente de la calidad de servicio y la conexión transparente del usuario no licenciado o de CR, cuando este transmite de forma oportunista en regiones licenciadas del espectro que no estén siendo usadas. Respondiendo a estas condiciones de diseño, la literatura divide una comunicación exitosa de CR en cuatro pasos, Detección, Decisión, Compartición y Movilidad.

El presente artículo se enfoca en la etapa de Detección de espectro para CR. En la primera parte se discuten conceptos básicos de detección y se brindan algunos ejemplos de las múltiples dimensiones que tiene el espectro electromagnético. La sección principal, seguida por las conclusiones, describe de forma jerárquica un número considerable de técnicas de estimación y detección de usuarios primarios con sus ventajas y desventajas.

\section{DETECCIÓN DE ESPECTRO- CONCEPTO BÁSICO}

La detección de espectro (Spectrum Sensing) ha sido brevemente definida como la labor de obtener un sentido de espectro disponible, determinando la existencia de usuarios licenciados dentro de una región geográfica definida. Este sentido se obtiene de diversas formas dentro de las cuales se encuentra la detección local del espectro en CR [3-5]. Un usuario de $\mathrm{CR}$ puede transmitir únicamente en frecuencias que no estén siendo usadas en el espectro que lo rodea. Por lo tanto, dicho usuario debe monitorear las bandas disponibles y capturar su información para finalmente detectar lo que se conoce como huecos espectrales [6].

A pesar de que la detección de espectro es tradicionalmente entendida como la medida de la energía de las frecuencias de radio a lo largo del espectro, cuando se trata de CR la detección se convierte en un término más amplio que involucra la obtención de características de uso espectral en múltiples dimensiones como el tiempo, el espacio, la frecuencia y el código [7]. La detección comprende una gran variedad de aspectos asociados con la determinación del tipo de señal que ocupa una banda; sin embargo, esto requiere técnicas más robustas para el análisis de señales, que suponen mayor complejidad desde el punto de vista computacional.

A continuación se presentan los conceptos asociados a las posibles dimensiones en las que se puede explotar el espectro para su uso oportunista por parte de los sistemas de CR.

\section{Consideración de las múltiples dimensiones del espectro}

Las dimensiones básicas de la Figura 1 constituyeron el modelo inicial para analizar la subutilización del espectro en los primeros estudios de radio cognitiva [8]; sin embargo, en estudios posteriores se plantearon nuevas dimensiones que hacen posible un uso aún más eficiente del espectro, teniendo en cuenta aspectos como los esquemas de codificación y el uso de antenas inteligentes. Estos dos aspectos se pueden interpretar como dos nuevas dimensiones para el uso oportunista del espectro. De este modo, y sumado a las dimensiones básicas ya mencionadas, se propone una detección de espectro disponible donde se incluyan las dimensiones de código y ángulo [7].

A continuación se proveen comentarios que aclaran las diferentes oportunidades de uso espectral asociadas a las cinco dimensiones discutidas en esta sección.

\section{Frecuencia y tiempo}

En este espacio bidimensional se buscan oportunidades en el dominio de la frecuencia. 
Las diferentes bandas de espectro disponible se dividen en porciones más angostas donde aparecen oportunidades espectrales, dado que no todas las bandas son usadas simultáneamente, Figura 1. Cada hueco espectral de este diagrama representa una oportunidad de transmisión para los dispositivos de $\mathrm{CR}$ en las dimensiones de frecuencia y tiempo. En [11], por ejemplo, se explotan los periodos ociosos entre las transmisiones por ráfaga de las señales en una red WLAN (Wireless Local Area Network).

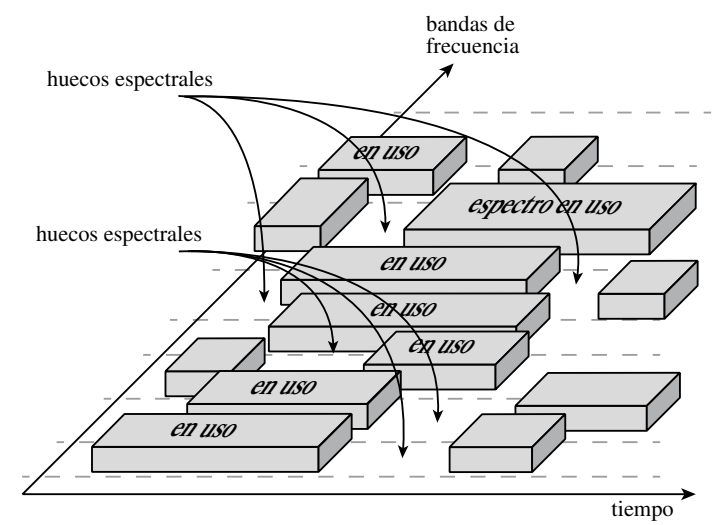

Figura 1. Dimensiones básicas tiempo y frecuencia.

\section{Espacio geográfico}

Aquí se buscan oportunidades con respecto a la ubicación (latitud, longitud, elevación) y a la distancia con respecto a los usuarios licenciados. Esto resulta de las pérdidas por propagación en el espacio. Estas medidas de propagación pueden ser evitadas mediante la observación de los niveles de interferencia. La Figura 2 muestra tres transmisiones simultáneas que pueden estar ocupando la misma banda de frecuencias sin generar interferencia entre ellas, debido a que los rangos de dichas transmisiones son menores a la distancia geográfica entre las mismas. Esta dimensión de oportunidades espectrales se relaciona directamente con el concepto de temperatura de interferencia establecido por la FCC (Comisión Federal de Comunicaciones) [1].

\section{Código}

En esta dimensión se busca detectar las secuencias de los códigos de espectro ensanchado, tanto de los saltos en tiempo (TH) como de los saltos en frecuencia $(\mathrm{FH})$ que son utilizadas por los usuarios licenciados. También se busca información de sincronización para que los usuarios de CR coordinen sus transmisiones con respecto a los usuarios

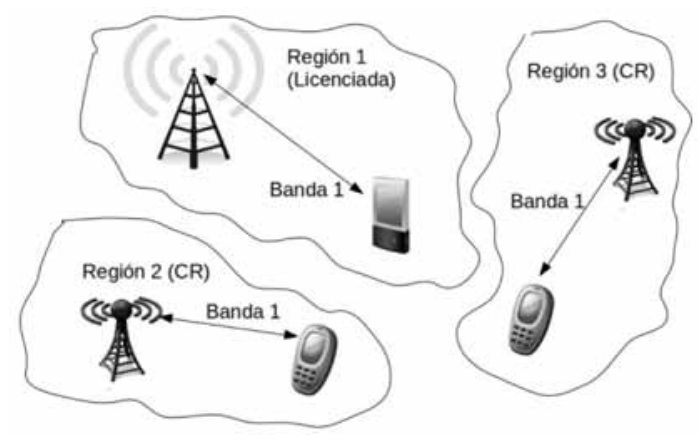

Figura 2. Transmisiones simultáneas en la misma banda de frecuencias explotando la dimensión espacio geográfico.

licenciados. La estimación de la sincronización puede ser evitada usando extensos códigos aleatorios; sin embargo, la interferencia parcial en este caso es inevitable.

El espectro sobre una banda ancha puede estar siendo utilizado en un tiempo dado por señales de espectro ensanchado o por señales que utilicen saltos de frecuencia. Esto no significa que no exista disponibilidad espectral en dicha banda. Debe ser posible efectuar transmisiones simultáneas sin interferir a los usuarios licenciados si se usan códigos que sean ortogonales a los códigos de los correspondientes usuarios licenciados. Estas implementaciones requieren el uso adecuado de la dimensión código, y posiblemente determinar los parámetros del desvanecimiento selectivo en frecuencia con el objetivo de reducir la interferencia al mínimo [53]. La Figura 3 muestra una pila de posibles códigos de transmisión sobre una sola banda donde existen señales de espectro ensanchado.

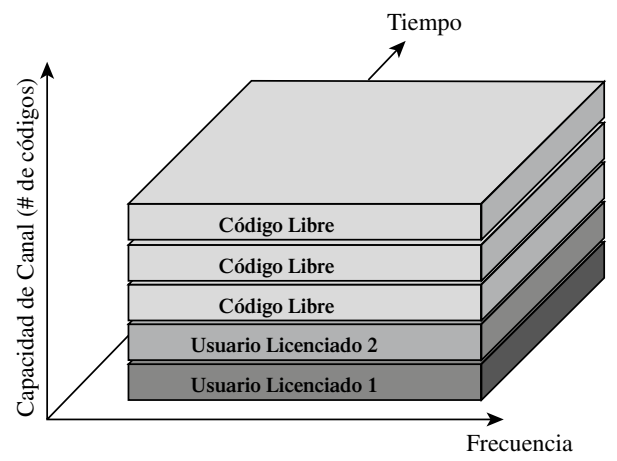

Figura 3. Representación de oportunidades de uso espectral en la dimensión código. 


\section{Ángulo}

En esta dimensión se detectan las direcciones de los lóbulos de radiación electromagnética (acimut y ángulo de elevación) y la ubicación de los usuarios licenciados. El conocimiento de la posición o dirección de los usuarios licenciados es lo que genera las oportunidades (los huecos) espectrales en la dimensión ángulo. La Figura 4 muestra diferentes transmisiones simultáneas que pueden usar la misma banda de frecuencia dentro de un mismo radio de alcance. Dado que las transmisiones son direccionales, el nivel de interferencia se reduce significativamente.

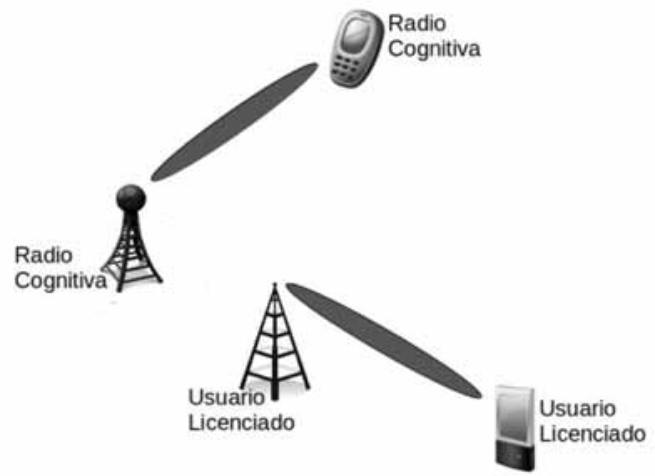

Figura 4. Transmisiones simultáneas dentro del mismo radio de alcance explotando el conocimiento de los ángulos de llegada de las señales.

Teniendo en cuenta las cinco dimensiones mencionadas, el espacio de ondas de radio puede ser definido como un hiperespacio teórico, ocupado por señales de radio, el cual tiene dimensiones de posición física, ángulo de llegada, frecuencia, tiempo y posiblemente otras más [9-10].

Los investigadores en CR sugieren el desarrollo de algoritmos avanzados para detección de espectro que incluyan estimación de oportunidades de uso espectral no-licenciado en múltiples dimensiones.

\section{RETOS DE LA DETECCIÓN DE USUARIOS LICENCIADOS}

\section{Requerimientos para los dispositivos físicos}

Es posible detectar el espectro a través de dos arquitecturas: arquitectura de radio única y arquitectura de radio dual [12-13]. En la arquitectura de radio única la eficiencia en el uso del espectro no es la ideal debido a que parte del tiempo de transmisión debe ser usado para detección y no para el envío de los datos [14-15]. La radio única asigna de manera constante un intervalo de tiempo para monitorear el espectro. Debido a esta restricción en el tiempo de detección, no es posible garantizar altos niveles de precisión al momento de estimar la ausencia de usuarios licenciados.

Por otro lado, en la arquitectura de radio dual existe un enlace dedicado para la transmisión y recepción de los datos. Otro enlace se encarga del monitoreo del espectro disponible [16-17]. Las desventajas de esta arquitectura son: el incremento en el consumo de energía y el costo de los equipos. Es pertinente mencionar que una sola antena sería suficiente para la conexión de ambos enlaces [12].

\section{Problema del usuario licenciado escondido}

Este problema es similar al problema del nodo escondido de los sistemas CSMA (Acceso Múltiple por Detección de Portadora). Esta situación puede ser ocasionada por varios factores, entre los cuales se encuentran el desvanecimiento (o sombreado) multicamino que experimentan los usuarios de CR al momento de detectar a los usuarios licenciados que se encuentren transmitiendo. En la Figura 5, el dispositivo de CR causa interferencia no-deseada sobre el usuario licenciado (el receptor) debido a que la señal del transmisor (estación licenciada) no pudo ser detectada dada su distancia con respecto a los usuarios de CR.

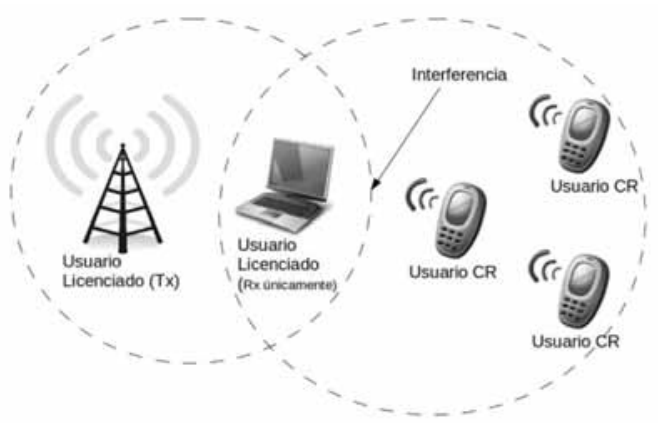

Figura 5. Problema del usuario escondido.

Para superar este problema se han propuesto esquemas de detección cooperativa [18-20]. 


\section{Detección de usuarios licenciados de espectro ensanchado}

Las tecnologías de transmisión inalámbrica para dispositivos comerciales pueden ser clasificadas de dos formas: tecnologías de frecuencia fija y tecnologías de espectro ensanchado. En las tecnologías de espectro ensanchado, como FHSS (Espectro Ensanchado por Salto de Frecuencia), los dispositivos cambian sus frecuencias de operación de forma dinámica ocupando múltiples canales de banda angosta. Estos cambios de frecuencia se conocen con el nombre de saltos y son realizados de acuerdo a una secuencia que debe ser conocida tanto por el transmisor como por el receptor.

Los usuarios licenciados que usan señalización de espectro ensanchado son difíciles de detectar debido a que su potencia de transmisión está distribuida sobre un amplio rango de frecuencias, a pesar de que su ancho de banda de información real sea mucho más angosto [21]. Este problema se puede evitar parcialmente si se conoce el patrón de los saltos y se logra una sincronización perfecta de las señales; sin embargo, diseñar algoritmos que puedan hacer una estimación apropiada en este espacio (dimensión) de códigos no es fácilmente alcanzable.

\section{Duración y frecuencia de las detecciones}

El número de veces que se debe detectar la disponibilidad del espectro en un intervalo de tiempo es un parámetro de diseño que debe ser considerado con detenimiento. El valor óptimo del tiempo entre detecciones depende de las capacidades del dispositivo de CR y de las características temporales de los usuarios licenciados en un determinado escenario [22]. En [23] se ha investigado el impacto que tienen los tiempos entre detecciones sobre el desempeño de los usuarios no licenciados. Con un enfoque similar, en [14], el tiempo de detección es obtenido utilizando optimización numérica, en este estudio la eficiencia del canal es maximizada para una determinada probabilidad de detección. En [24] se propone otro método donde el intervalo de guarda entre símbolos de Multiplexación por División de Frecuencia Ortogonal (OFDM) es reemplazado por periodos de inactividad y las detecciones se realizan en estos periodos inactivos.

Los tiempos de detección pueden ser reducidos al detectar solamente las partes del espectro que presenten cambios. Un método de detección que adapta los parámetros de barrido espectral de acuerdo a un modelo estimado de ocupación de canal es desarrollado en [25].

Un canal ocupado por usuarios no licenciados no puede ser empleado simultáneamente para hacer detecciones. Esta es la razón por la que los usuarios no licenciados deben interrumpir sus transmisiones de datos para realizar detecciones de espectro [24]. Estas interrupciones reducen la eficiencia espectral de todo el sistema [22]. Para minimizar este problema se propone un método llamado salto dinámico de frecuencia (DFH) [26].

Estos retos han conducido al desarrollo de diferentes técnicas y esquemas para la detección de usuarios licenciados, las cuales son cada vez más elaboradas; sin embargo, es importante mencionar que algunas de las técnicas más robustas sugieren una complejidad computacional y un consumo de energía que implicarían altos costos en la construcción de los dispositivos, o que simplemente serían muy difíciles de llevar a la práctica. A continuación se explican los conceptos que soportan a las técnicas existentes.

\section{TÉCNICAS DE DETECCIÓN DE ESPECTRO PARA RADIO COGNITIVA}

\section{Técnicas fundamentales}

Las métricas y los principios involucrados en estos tres modelos representan la información fundamental que posteriormente sirve de base para desarrollar algoritmos de detección más elaborados.

\section{Detección de energía}

La detección de energía en las bandas de frecuencia es la forma más común de detectar oportunidades en el espectro, debido a su bajo nivel de complejidad en términos de computación e implementación [13, 17-21, 23, 25, 27-46].

Considerando que la señal que se desea recibir es de la forma:

$$
y(n)=x(n)+r(n)
$$

En la ecuación (1), $x(n)$ es la señal objetivo, $r(n)$ es el ruido blanco gaussiano y $n$ es el índice de la muestra (en el dominio del tiempo) o el índice del símbolo (en el dominio de la frecuencia). Por simplicidad, se asumirá que las diferentes muestras de $x(n)$ son independientes. La correlación entre 
las muestras de $x(n)$ mejorará el desempeño de la detección. Debido a que las muestras de ruido $r(n)$ son también independientes, las muestras $y(n)$ recibidas serán independientes.

La métrica $M$ de la detección de energía corresponderá a la suma de las muestras detectadas en una banda del espectro en particular de la siguiente forma:

$$
M=\sum_{n=1}^{N}|y(n)|^{2}
$$

En la ecuación (2), $N$ es el tamaño del vector de muestras o número de muestras de la señal que caben en el buffer del detector de usuarios licenciados. Se debe notar que $|y(n)|^{2}$ es una secuencia de variables aleatorias independientes y distribuidas de forma idéntica con media $\mu$ y varianza $\sigma^{2}$.

Cuando $N$ es grande, usando el teorema del límite central, la métrica de detección $M$ puede ser aproximada como una variable aleatoria gaussiana con media $\mu_{M}=N$. $\mu$ y varianza $\sigma_{M}^{2}=N . \sigma^{2}$.

Nótese que $x(n)=0$ cuando no hay transmisiones por parte de los usuarios licenciados. De acuerdo a esta técnica, se determinará que existe un usuario licenciado transmitiendo en una banda determinada cuando la métrica $M$ supere un umbral específico $\lambda$. Esto es lo mismo que tomar una decisión con respecto a la siguiente hipótesis binaria:

$$
\begin{gathered}
H_{0}: y(n)=r(n) \\
H_{l}: y(n)=x(n)+r(n)
\end{gathered}
$$

De acuerdo a la decisión entre estas dos hipótesis, los índices más relevantes para este tipo de detección son las probabilidades de detección acertada $P_{D}$ y la probabilidad de falsa alarma $P_{F}$. La probabilidad $P_{D}$ equivale a decidir acertadamente que existe un usuario, es decir:

$$
P_{D=} P\left(M>\lambda \mid H_{1}\right)
$$

La probabilidad de falsa alarma $P_{F}$ es la probabilidad de perder oportunidades de uso espectral, dado que la métrica $M$ sobrepasa el umbral $\lambda$, cuando realmente no existen transmisiones de usuarios licenciados, es decir:

$$
P_{F}=P\left(M>\lambda \mid H_{0}\right)
$$

Lo que se busca es un balance adecuado entre $P_{F}$ y $P_{D}$ para obtener un umbral $\lambda$ óptimo.

El principal atractivo de esta técnica es que los receptores de Radio Cognitiva no requieren ningún conocimiento de la señal del usuario primario. Las señales son detectadas comparando el nivel de salida de un detector de energía con respecto a un umbral que depende del ruido [47, 57]. Sin embargo, la selección de este umbral es una de las debilidades de esta técnica. Otra falencia de la detección de energía es la falta de precisión al diferenciar el ruido de la interferencia producida por otros usuarios licenciados. Adicionalmente, esta técnica tiene un desempeño limitado cuando se aplica en condiciones de baja relación señal a ruido y cuando se desea detectar señales de espectro ensanchado [21, 42].

El umbral que se usa en los algoritmos de detección de energía depende de la varianza del ruido, por este motivo, un pequeño error en la estimación de la potencia del ruido produce una baja significativa en el desempeño [48]. Para solucionar este problema, el nivel de ruido es estimado de forma dinámica mediante el uso de algoritmos de clasificación de señal múltiple (MUSIC) [49]. En [45] se propone un algoritmo iterativo para hallar el umbral. Dicho umbral debe satisfacer cierto nivel de confianza, por ejemplo una probabilidad de falsa alarma determinada. Otras metodologías más recientes basadas en detección de energía son estudiadas bajo condiciones de potencia de ruido desconocida [37], donde se propone estimar los niveles de ruido de forma adaptativa.

\section{Detección por filtro adaptado}

Básicamente un filtro adaptado adecua su respuesta al impulso de manera tal que al tomar las muestras de la señal en un tiempo específico, la salida del filtro sea equivalente a la salida de un receptor de correlación. Esta técnica es reconocida por su óptimo desempeño en la detección de usuarios licenciados cuando las señales transmitidas son conocidas [50]. Este tipo de filtrado logra ciertas probabilidades de falsa alarma y de detección errónea en tiempos considerablemente cortos [51]. Sin embargo, la detección por filtro adaptado hace necesario que el receptor de CR demodule las señales recibidas, lo cual requiere un conocimiento 
muy alto de las características de las señales de los usuarios licenciados (ancho de banda, frecuencia de operación, tipo y orden de modulación, formado de los pulsos y formato de las tramas). La complejidad de implementar una unidad de detección con estas características es muy alta para ser llevada a la práctica [21].

\section{Detección basada en características cicloesta- cionarias}

En este caso, para detectar la presencia de usuarios licenciados se explota la naturaleza periódica de las señales moduladas, es decir, que a diferencia de lo que sucede en la detección de energía, una señal que porte información será muy diferente del ruido y de la interferencia debido a los cambios consecutivos que se presentan en periodos iguales de tiempo, e.g. la tasa a la que cambian los símbolos o la periodicidad intrínseca de las mismas portadoras. Desde el punto de vista formal, un proceso estocástico $X(t)$ que represente a una señal modulada linealmente con respecto a una secuencia de símbolos (o de bits) se puede representar como:

$$
X(t)=\sum_{n=-\infty}^{\infty} a_{n} g(t-n T)
$$

En la ecuación (7), $\left\{a_{n}\right\}$ es una secuencia de variables aleatorias (en tiempo discreto) con media $\mu_{a}=E\left[a_{n}\right]$ para todo $n$ y la secuencia de autocorrelación $C_{a a}(k)=E\left[a \cdot a_{n+k} *\right]$. La señal $g(t)$ es determinística (i.e. las portadoras). La secuencia $\left\{a_{n}\right\}$ representa la secuencia de símbolos digitales transmitidos por el canal, cuya tasa de transmisión es $1 / T$. Dado este periodo $T$ para el cambio de un símbolo a otro, se puede demostrar fácilmente que la secuencia de correlación de todo el proceso estocástico, es decir $C_{x x}(t, t+\tau)$, es periódica:

$$
\begin{aligned}
C_{x x}(t, t+\tau) & =E[X(t) X *(t+\tau)] \\
& =C_{x x}(t+k T, t+\tau+k T)
\end{aligned}
$$

$\mathrm{Al}$ ser periódica, $C_{x x}$ puede ser representada por una serie de Fourier con frecuencia fundamental $\alpha$ de la siguiente forma:

$$
C_{x x}(t, t+\tau)=\sum_{\alpha} C_{x x}^{\alpha}(\tau) e^{j 2 \pi \alpha t}
$$

Esta sumatoria recorre los múltiplos enteros de la frecuencia fundamental $\alpha$. Los coeficientes de esta serie, los cuales dependen del retraso $\tau$, pueden ser calculados como:

$$
C_{x x}^{\alpha}(\tau)=\lim _{T \rightarrow \infty} \frac{1}{T} \int_{-T / 2}^{T / 2} C_{x x}(t, t+\tau) e^{-j 2 \pi \alpha t} d t
$$

La función $C_{x x}^{\alpha}(\tau)$ es conocida como función de autocorrelación cíclica. La densidad de correlación espectral $S_{x x}^{\alpha}(f)$ se define como la transformada de Fourier de $C_{x x}^{\alpha}(\tau)$ :

$$
S_{x x}^{\alpha}(f)=\int_{-\infty}^{\infty} C_{x x}^{\alpha}(\tau) e^{-j 2 \pi f \tau} d \tau
$$

Esta expresión puede ser vista como una generalización de la función convencional de densidad espectral de potencia. Una modificación muy útil de la función $C_{x x}^{\alpha}(\tau)$ de la ecuación (10) es su función conjugada, definida como:

$$
C_{x x^{*}}^{\alpha}(\tau)=\lim _{T \rightarrow \infty} \frac{1}{T} \int_{-T / 2}^{T / 2} C_{x x^{*}}(t, t+\tau) e^{-j 2 \pi \alpha t} d t
$$

En la ecuación (12) $C_{x x^{*}}(t, t+\tau)=E[X(t) X(t+\tau)]$. Igual que en el caso no-conjugado, la función de densidad de correlación espectral conjugada $S_{x x^{*}}^{\alpha}(f)$ es la transformada de Fourier de $C_{x x^{*}}^{\alpha}(\tau)$. Las funciones $C_{x x}^{\alpha}(\tau)$ y $C_{x x^{*}}^{\alpha}(\tau)$ son discretas con respecto a la frecuencia fundamental $\alpha \mathrm{y}$ continuas con respecto a $\tau$. En una señal que no tenga características cicloestacionarias $C_{x x}^{\alpha}(\tau)=0$ y $C_{x x^{*}}^{\alpha}(\tau)=0 \forall \alpha \neq 0$. Los valores de $\alpha$, para los cuales estas dos funciones son diferentes de cero, se encuentran en las periodicidades ocultas del proceso estocástico $X(t)$ de la ecuación (7). Estos valores de $\alpha$ representan marcas cíclicas inherentes a la señal detectada. Por lo tanto, para un dispositivo de CR existe mayor certeza al diferenciar una verdadera señal de información de la interferencia producida por las señales de otros usuarios licenciados y de la varianza del ruido.

Las características cicloestacionarias también pueden ser inducidas intencionalmente para contribuir con la detección del espectro [61-63]. Los algoritmos empleados en esta técnica diferencian el ruido de las señales de los usuarios licenciados. Lo anterior se logra debido a que el ruido es estacionario (WSS) y sin correlación alguna, mientras que las señales moduladas son cicloestacionarias [54]. Adicionalmente, las características cicloestacionarias pueden ser usadas para distinguir entre diferentes tipos de modulación y de usuarios licenciados [58], 
[60]. Se puede asumir que las frecuencias cíclicas son conocidas $[52,56]$; en caso contrario, estas pueden ser extraídas y usadas como características para identificar las señales transmitidas [55]. La forma de onda de las señales OFDM es alterada antes de ser transmitida [61-63] con el objetivo de generar marcas específicas del sistema en ciertas bandas. Estas marcas son usadas para proporcionar un mecanismo efectivo de clasificación de señales. En [63], se eleva el número de características generadas en la señal para hacer el sistema más robusto ante el desvanecimiento multicamino. La desventaja en este caso es la pérdida de ancho de banda, debida al aumento de bits que no portan información de la señal en la transmisión. A pesar de que los métodos en [61] y [62] hayan sido propuestos específicamente para OFDM, se pueden desarrollar técnicas similares para cualquier tipo de señal [64]. En [65] se presenta una implementación de hardware para un detector de características cicloestacionarias.

\section{Evolución de las técnicas fundamentales}

En la mayoría de los casos, el principio básico es la detección de energía, seguida por la extracción de características cicloestacionarias. En otros ejemplos, sin llegar a utilizar un filtro adaptado, lo que se busca es tener tanta información de la señal como sea posible para efectuar procesos de correlación de señales y/o vectores.

\section{Detección de forma de onda (detección coherente)}

El uso de patrones es normalmente implementado en los sistemas inalámbricos para diferentes propósitos, e.g. sincronización. Dichos patrones incluyen el uso de preámbulos, patrones de mitad de trama, patrones piloto transmitidos regularmente, secuencias de espectro ensanchado, entre otros. Cada vez que sucede un patrón conocido, se puede efectuar una detección al correlacionar la señal recibida con una copia conocida de sí misma [31, 41], 46]. Con respecto a la métrica empleada para tomar la decisión de si existe o no un usuario licenciado al recibir la señal de la ecuación (1), un detector de forma de onda calculará el producto de los vectores $y(n), x(n)$ como se muestra a continuación:

$$
\begin{aligned}
M & =\operatorname{Re}\left[\sum_{n=1}^{N} y(n) x *(n)\right] \\
& =\sum_{n=1}^{N}|x(n)|^{2}+\operatorname{Re}\left[\sum_{n=1}^{N} r(n) x *(n)\right]
\end{aligned}
$$

Esto hace que el umbral $\lambda$ con el que se compara $M$ sea más fácil de determinar que en el caso de la detección de energía. Este método es solamente aplicable a los sistemas que involucran señales con patrones conocidos. En [31] se demuestra que este tipo de detección tiene mejor desempeño que la detección de energía en términos de confiabilidad y tiempo de convergencia. En [38, 39] se explotan los preámbulos de paquete de las señales IEEE $802.11 \mathrm{~b}$ [66] para analizar las características de uso de un canal WLAN. Los resultados de las mediciones presentados en [20] muestran cómo la detección basada en Forma de Onda emplea tiempos de medida muy cortos. La desventaja de este tipo de detección está en los errores de sincronización que se puedan presentar. Los preámbulos de paquete también son explotados para detectar señales WiMAX [46].

\section{Detección por identificación del radiotransmisor} Es posible obtener un conocimiento completo de las características del espectro al identificar la tecnología de transmisión que está siendo usada por los usuarios licenciados. Este tipo de identificación permite mayor precisión [42]. Adicionalmente, los sistemas de CR podrían comunicarse con las tecnologías identificadas para aplicaciones específicas. En el contexto del proyecto europeo llamado TRUST (Transparent Ubiquitous Terminal) [67], se emplean técnicas de clasificación y extracción de características para efectuar detecciones de espectro usando identificación de radio.

El procedimiento en estas técnicas es básicamente la extracción de un número significativo de características de las señales recibidas, seguido de la implementación de varios métodos de clasificación. Las características obtenidas de la detección de energía son usadas para efectuar la clasificación correspondiente $[12,68]$. Dicha extracción incluye la cantidad de energía detectada y su distribución espectral. El ancho de banda del canal y su forma también son usados como características de referencia [69], en este estudio la clasificación se hizo con redes neuronales. En [42], el ancho de banda de operación y la frecuencia central de la señal recibida se extraen usando métodos basados en detección de energía. Estas dos características son procesadas por un clasificador bayesiano para determinar los usuarios licenciados activos. La desviación estándar de la frecuencia instantánea y la duración máxima de la señal son extraídas usando 
análisis de tiempo-frecuencia para posteriormente emplear redes neuronales en la identificación de transmisiones activas [70-73]. En [74] se utilizan las características cicloestacionarias de las señales para obtener la información de los canales vacantes de tecnología GSM de un usuario licenciado. En [59] las frecuencias cíclicas de las señales de entrada son utilizadas tanto para la detección como para la clasificación de las señales, aquí la identificación de las señales se efectúa mediante el procesamiento de las características cicloestacionarias usando Modelos Escondidos de Markov (HMM). Otro método basado en características cicloestacionarias es implementado en $[52,55]$, donde se usan como características la densidad de correlación espectral y la función de coherencia espectral. En la etapa de clasificación, se usan redes neuronales en [55] y pruebas estadísticas en [52].

\section{Detección espectral multitaper}

Este tipo de detección es una aproximación del estimador de máxima verosimilitud para Densidad Espectral de Potencia [6]. El algoritmo se acerca mucho a una solución óptima para señales de espectro ensanchado. A pesar de que la complejidad de este método es menor que la del estimador de máxima verosimilitud, este método sigue siendo exigente en términos de carga computacional.

\section{Detección basada en transformada Wavelet}

En este caso se usan Wavelets para detectar los bordes de la densidad espectral de potencia de un canal de banda ancha [75], el espectro de frecuencia puede ser caracterizado como ocupado o vacío (de forma binaria) usando la información de la potencia hallada y de los bordes. En [76] se extiende el método propuesto en [75] usando un muestreo similar al de Nyquist. La implementación análoga de la detección basada en transformada Wavelet está sugerida en $[16,77-78]$, para efectuar detecciones no detalladas. La detección de Espectro con Multiresolución se logra mediante el cambio de las funciones base sin necesidad de modificar los circuitos de detección [16]. Las funciones base se cambian ajustando el ancho de pulso de las Wavelets y la frecuencia portadora. De este modo es posible realizar una detección rápida al enfocarse en las frecuencias con transmisiones activas después de hacer un barrido superficial. En [78] se explica la implementación de un banco de prueba para este algoritmo.

\section{Detección basada en transformada Hough}

La transformada Hough Aleatoria de la señal recibida es usada para identificar la presencia de pulsos de radar [79] en los canales de operación de los sistemas IEEE 802.11. Este método también puede ser usado para detectar cualquier tipo de señales con un patrón periódico. Se sabe que la covarianza estadística del ruido y de la señal son diferentes. Esta propiedad es aprovechada para desarrollar algoritmos [80] que identifican la existencia de una señal de comunicaciones. En este estudio se muestra que los métodos propuestos son efectivos en la detección de señales de televisión digital.

\section{Detección de la potencia de fuga de los osciladores locales}

Los receptores de radio modernos se basan en su gran mayoría en la arquitectura de receptor superheterodino, ver Figura 6.

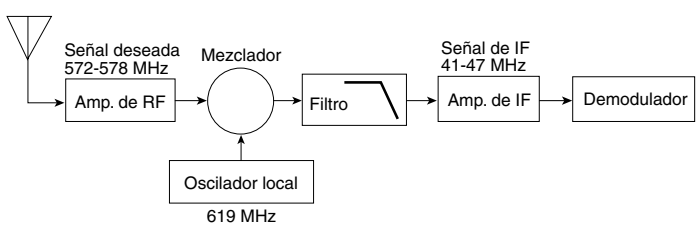

Figura 6. Receptor superheterodino.

Esta arquitectura convierte la señal de RF en una señal de frecuencia intermedia (IF), permitiendo reemplazar un filtro RF ajustable con un factor $\mathrm{Q}$ bajo por un filtro de frecuencia intermedia menos costoso que posee un factor Q muy superior. Para convertir la señal de RF a la frecuencia intermedia (IF) se debe utilizar un Oscilador Local, cuya potencia es reflejada hacia la antena y por lo tanto es irradiada. Esta radiación mínima emitida por estos osciladores locales puede ser utilizada para detectar a los receptores de las frecuencias licenciadas.

A diferencia de otras técnicas de detección que centran su atención en buscar a los transmisores licenciados, en este caso se detectan oportunidades de uso del espectro buscando a los usuarios licenciados que están recibiendo las señales dentro del rango de comunicación de un usuario de CR. En [81] se explota la potencia emitida por los osciladores locales. Pese a que esta técnica permite identificar a los receptores licenciados, la potencia de referencia es tan baja que la implementación de un detector confiable no es sencilla. En la actualidad este método 
solamente ha sido llevado a la práctica para detectar receptores de televisión.

\section{CONCLUSIONES}

En este artículo se describieron las técnicas utilizadas para detectar usuarios licenciados por parte de los dispositivos de CR. Adicionalmente, se aclaró el concepto de espectro multidimensional. Las técnicas expuestas están siendo estudiadas para garantizar interferencia mínima sobre los usuarios licenciados, ofreciendo un uso eficiente de canales que soporten los anchos de banda de las aplicaciones actuales (calidad de servicio para datos y multimedia). Una detección adecuada es el punto de partida para el correcto funcionamiento de las demás etapas del ciclo cognitivo en los sistemas de CR (decisión, compartición y movilidad). En la actualidad la mayoría de estudios están utilizando técnicas de detección basadas en energía y en características cicloestacionarias. Gran parte de los retos asociados a aspectos como la incertidumbre con respecto al ruido, el desvanecimiento y los terminales ocultos se pueden resolver empleando esquemas de detección cooperativa. Las detecciones cooperativas reducen en gran medida la probabilidad de detección errada y de falsas alarmas. Adicionalmente, la cooperación entre nodos de CR puede resolver el problema del terminal escondido y reducir los tiempos de detección [18-20]. En general, las dimensiones del espectro explotadas se limitan en su mayoría a espacio, frecuencia y rango. Estudios futuros deberían enfocarse en el análisis de otro tipo de técnicas (o de técnicas híbridas). En este sentido, se deben estudiar nuevas oportunidades de uso eficiente del espectro que involucren más activamente a las dimensiones espectrales asociadas a los códigos de las señales y sus ángulos de llegada.

\section{REFERENCIAS}

[1] Federal Communications Commission. "Notice of proposed rule making and order". ET Docket No 03-322. 2003.

[2] I.F. Akyildiz, W.-Y. Lee, M. C. Vuran and Sh. Mohanty. "A survey on spectrum management in cognitive radio networks". IEEE Communications Magazine. 2008.

[3] Federal Communications Commission. "Notice of proposed rule making: Unlicensed operation in the tv broadcast bands". ET Docket No. 04-186 (FCC 04-113). 2004.

[4] M. Marcus. Unlicensed cognitive sharing of tv spectrum: "The controversy at the federal communications commission". IEEE Commun. Mag. Vol. 43, Issue 5, pp. 24-25. 2005.

[5] Y. Zhao, L. Morales, J. Gaeddert, K.K. Bae, J.-S. Um and J.H Reed. "Applying radio environment maps to cognitive wireless regional area networks". Proc. IEEE Int. Symposium on New Frontiers in Dynamic Spectrum Access Network, pp. 115-118. 2007.

[6] S. Haykin. "Cognitive radio: Brain-empowered wireless communications". IEEE J. Select. Areas Commun. Vol. 23, Issue 2, pp. 201220. February, 2005.

[7] T. Yucek and H. Arslan. "A survey of spectrum sensing algorithms for cognitive radio applications". IEEE Communications survey \& tutorials. Vol. 11, Issue 1. 2009.

[8] P. Kolodzy. "Next generation communications: Kickoff meeting". In Proc. DARPA. 2001.

[9] R. Matheson. "The electrospace model as a frequency management tool". In Int. Symposium on Advanced Radio Technologies, pp. 126-132. 2003.

[10] A.L. Drozd, I.P. Kasperovich, C.E. Carroll and A.C. Blackburn. "Computational electromagnetics applied to analyzing the efficient utilization of the $\mathrm{rf}$ transmission hyperspace". In Proc. IEEE/ACES Int. Conf. on Wireless Communications and Applied Computational Electromagnetics, pp. 1077 1085. 2005.

[11] S. Geirhofer, L. Tong and B. Sadler. "Dynamic spectrum access in the time domain: Modeling and exploiting white space". IEEE Commun. Mag. Vol. 45, Issue 5, pp. 66-72. 2007.

[12] G. Vardoulias, J. Faroughi-Esfahani, G. Clemo and R. Haines. "Blind radio access technology discovery and monitoring for software defined radio communication systems: problems and techniques". In Proc. Int. Conf. 3G Mobile Communication Technologie, pp. 306-310. 2001.

[13] S. Shankar, C. Cordeiro and K. Challapali. "Spectrum agile radios: utilization and sensing architectures". In Proc. IEEE Int. Symposium 
on New Frontiers in Dynamic Spectrum Access Networks, pp. 160-169. 2005.

[14] P. Wang, L. Xiao, S. Zhou and J. Wang. "Optimization of detection time for channel efficiency in cognitive radio systems". In Proc. IEEE Wireless Commun. and Networking Conf., pp. 111-115. 2007.

[15] Q. Zhao, S. Geirhofer, L. Tong and B.M. Sadler. "Optimal dynamic spectrum access via periodic channel sensing". In Proc. IEEE Wireless Commun. and Networking Conf., pp. 33-37. 2007.

[16] Y. Hur, J. Park, W. Woo, K. Lim, C. Lee, H. Kim and J. Laskar. "A wideband analog multi-resolution spectrum sensing (MRSS) technique for cognitive radio (CR) systems". In Proc. IEEE Int. Symp. Circuits and Systems, pp. 4090-4093. 2006.

[17] Y. Yuan, P. Bahl, R. Chandra, P. A. Chou, J. I. Ferrell, T. Moscibroda, S. Narlanka and Y. Wu. "Knows: Cognitive radio networks over white spaces". Symposium on New Frontiers in Dynamic Spectrum Access Networks, pp. 416-427. 2007.

[18] G. Ganesan and Y. Li. "Agility improvement through cooperative diversity in cognitive radio". In Proc. IEEE Global Telecomm. Conf. (Globecom). Vol. 5, pp. 2505-2509. 2005.

[19] G. Ganesan and Y. Li. "Cooperative spectrum sensing in cognitive radio networks". Proc. IEEE Int. Symposium on New Frontiers in Dynamic Spectrum Access Networks, pp. 137-143. 2005.

[20] D. Cabric, A. Tkachenko and R. Brodersen. "Spectrum sensing measurements of pilot, energy, and collaborative detection". In Proc. IEEE Military Commun. Conf., pp. 1-7. 2006.

[21] D. Cabric, S. Mishra and R. Brodersen. "Implementation issues in spectrum sensing for cognitive radios". In Proc. Asilomar Conf. on Signals, Systems and Computers. Vol. 1, pp. 772-776. 2004.

[22] S.D. Jones, E. Jung, X. Liu, N. Merheb and I.-J. Wang. "Characterization of spectrum activities in the u.s. public safety band for opportunistic spectrum access". In Proc. IEEE Int. Symposium on New Frontiers in Dynamic Spectrum Access Networks, pp. 137-146. 2007.
[23] A. Ghasemi and E. Sousa. "Optimization of spectrum sensing for opportunistic spectrum access in cognitive radio networks". In Proc. IEEE Consumer Commun. and Networking Conf., pp.1022-1026. 2007.

[24] N. Khambekar, L. Dong and V. Chaudhary. "Utilizing ofdm guard interval for spectrum sensing". In Proc. IEEE Wireless Commun. and Networking Conf., pp. 38-42. 2007.

[25] D. Datla, R. Rajbanshi, A.M. Wyglinski and G.J. Minden. "Parametric adaptive spectrum sensing framework for dynamic spectrum access networks". In Proc. IEEE Int. Symposium on New Frontiers in Dynamic Spectrum Access Networks, pp. 482-485. 2007.

[26] W. Hu, D. Willkomm, M. Abusubaih, J. Gross, G. Vlantis, M. Gerla and A. Wolisz. "Dynamic frequency hopping communities for efficient ieee 802.22 operation". IEEE Commun. Mag. Vol. 45, Issue 5, pp. 80-87. 2007.

[27] T. Weiss, J. Hillenbrand and F. Jondral. "A diversity approach for the detection of idle spectral resources in spectrum pooling systems". In Scientific Colloquium, pp. 37-38. 2003.

[28] F. Digham, M. Alouini and M. Simon. "On the energy detection of unknown signals over fading channels". In Proc. IEEE Int. Conf. Commun. Vol. 5, pp. 3575-3579. 2003.

[29] P. Qihang, Z. Kun, W. Jun and L. Shaoqian. "A distributed spectrum sensing scheme based on credibility and evidence theory in cognitive radio context". In Proc. IEEE Int. Symposium on Personal, Indoor and Mobile Radio Commun, pp. 1-5. 2006.

[30] P. Pawelczak, G.J. Janssen and R.V. Prasad. "Performance measures of dynamic spectrum access networks". In Proc. IEEE Global Telecomm. Conf. (Globecom). 2006.

[31] H. Tang. "Some physical layer issues of wide-band cognitive radio systems". In Proc. IEEE Int. Symposium on New Frontiers in Dynamic Spectrum Access Networks, pp.151-159. 2005.

[32] M. Wylie-Green. "Dynamic spectrum sensing by multiband ofdm radio for interference mitigation". In Proc. IEEE Int. Symposium on New Frontiers in Dynamic Spectrum Access Networks, pp. 619-625. 2005. 
[33] S. Jones and N. Wang. "An experiment for sensing-based opportunistic spectrum access in csma/ca networks". In Proc. IEEE Int. Symposium on New Frontiers in Dynamic Spectrum Access Networks, pp. 593-596. 2005.

[34] P. Papadimitratos, S. Sankaranarayanan and A. Mishra. "A bandwidth sharing approach to improve licensed spectrum utilization". IEEE Commun. Mag. Vol. 43, Issue 12, pp. 10-14. 2005.

[35] A. Leu, K. Steadman, M. McHenry and J. Bates. "Ultra sensitive TV detector measurements". In Proc. IEEE Int. Symposium on New Frontiers in Dynamic Spectrum Access Networks, pp. 30-36. 2005.

[36] J. Lehtom Ki. "Analysis of energy based signal detection". $\mathrm{PhD}$ thesis. University of Oulu. 2005.

[37] J. Lehtom Ki, J. Vartiainen, M. Juntti and H. Saarnisaari. "Spectrum sensing with forward methods". In Proc. IEEE Military Commun. Conf., pp.1-7. 2006.

[38] S. Geirhofer, L. Tong and B. Sadler. "A measurement-based model for dynamic spectrum access in wlan channels". In Proc. IEEE Military Commun. Conf. 2006.

[39] S. Geirhofer, B. Sadler and L. Tong. "Dynamic spectrum access in wlan channels: Empirical model and its stochastic analysis". In Proc. of Int. Workshop on Technology and Policy for Accessing Spectrum. 2006.

[40] A.E. Leu, M. McHenry and B.L. Mark. "Modeling and analysis of interference in listen-before-talk spectrum access schemes". Int. Journal of Network Management. Vol. 16, Issue 2, pp.131-147. 2006.

[41] A. Sahai, R. Tandra, S.M. Mishra and N. Hoven. "Fundamental design tradeoffs in cognitive radio systems". In Proc. of Int. Workshop on Technology and Policy for Accessing Spectrum. 2006.

[42] T.Y Cek and H. Arslan. "Spectrum characterization for opportunistic cognitive radio systems". In Proc. IEEE Military Commun. Conf., pp. 1-6. 2006.

[43] P. Pawełczak, C. Guo, R. Prasad and R. Hekmat. "Cluster-based spectrum sensing architecture for opportunistic spectrum access networks". Tech. Rep. IRCTR-S-004-07. 2007.
[44] X. Liu and S. Shankar. "Sensing-based opportunistic channel access". Mobile Networks and Applications. Vol. 11, Issue 4, pp. 577-591. 2006.

[45] F. Weidling, D. Datla, V. Petty, P. Krishnan and G. Minden. "A framework for $r$ spectrum measurements and analysis". In Proc. IEEE Int. Symposium on New Frontiers in Dynamic Spectrum Access Networks. Vol. 1, pp. 573576. 2005.

[46] Sh.M. Mishra, S.T. Brink, R. Mahadevappa and R.W. Brodersen. "Cognitive technology for ultra-wideband/wimax coexistence". In Proc. IEEE Int. Symposium on New Frontiers in Dynamic Spectrum Access Networks, pp. 179-186. 2007.

[47] H. Urkowitz. "Energy detection of unknown deterministic signals". Proc. IEEE. Vol. 55, pp. 523-531. 1967.

[48] N. Hoven, A. Sahai and R. Tandra. "Some fundamental limits on cognitive radio". In Proc. Allerton Conf. on Commun Control, and Computing. 2004.

[49] M.P. Olivieri, G. Barnett, A. Lackpour and A. Davis. "A scalable dynamic spectrum allocation system with interference mitigation for teams of spectrally agile software defined radios". In Proc. IEEE Int. Symposium on New Frontiers in Dynamic Spectrum Access Networks, pp. 170-179. 2005.

[50] J.G. Proakis. "Digital Communications" McGraw-Hill (editorial), 4th edition. 2001.

[51] R. Tandra and A. Sahai. "Fundamental limits on detection in low snr under noise uncertainty". In Proc. IEEE Int. Conf. Wireless Networks, Commun. and Mobile Computing. Vol. 1, pp. 464-469. 2005.

[52] M. Oner and F. Jondral. "Cyclostationarity based air interface recognition for software radio systems". In Proc. IEEE Radio and Wireless Conf., pp. 263-266. 2004.

[53] L. Pedraza, C. Hernández and D. Ballesteros. "Modelo del desvanecimiento selectivo en frecuencia". Revista Fac. de Ing. Univ. Antioquia. $N^{\circ}$ 58, pp. 114-122. 2011.

[54] D. Cabric and R.W. Brodersen. "Physical layer design issues unique to cognitive radio systems". In Proc. IEEE Int. Symposium on Personal, Indoor and Mobile Radio Commun. Vol. 2, pp. 759-763. 2005. 
[55] A. Fehske, J. Gaeddert and J. Reed. "A new approach to signal classification using spectral correlation and neural networks". In Proc. IEEE Int. Symposium on New Frontiers in Dynamic Spectrum Access. Networks, pp. 144-150. 2005.

[56] M. Ghozzi, F. Marx, M. Dohler and J. Palicot. "Cyclostationarity-based test for detection of vacant frequency bands". In Proc. IEEE Int. Conf. Cognitive Radio Oriented Wireless Networks and Commun. 2006.

[57] R Ferro, L. Pedraza and C. Hernández. "Maximización del Throughput en una red de radio cognitiva basado en la probabilidad de falsa alarma". Tecnura. Vol. 15, $\mathrm{N}^{\circ} 30$, pp. 64-70. 2011.

[58] J. Lundn, V. Koivunen, A. Huttunen and H.V. Poor. "Spectrum sensing in cognitive radios based on multiple cyclic frequencies". In Proc. IEEE Int. Conf. Cognitive Radio Oriented Wireless Networks and Commun. 2007.

[59] K. Kim, I.A. Akbar, K.K. Bae, J.-S. Um, C.M. Spooner and J.H. Reed. "Cyclostationary approaches to signal detection and classification in cognitive radio". In Proc. IEEE Int. Symposium on New Frontiers in Dynamic Spectrum Access Networks, pp. 212-215. 2007.

[60] W.A. Gardner. "Exploitation of spectral redundancy in cyclostationary signals". IEEE Signal Processing Mag. Vol. 8, Issue 2, pp.14-36. 1991.

[61] K. Maeda, A. Benjebbour, T. Asai, T. Furuno and T. Ohya. "Recognition among ofdm-based systems utilizing cyclostationarity-inducing transmission". In Proc. IEEE Int. Symposium on New Frontiers in Dynamic Spectrum Access Networks, pp. 516-523. 2007.

[62] P.D. Sutton, K.E. Nolan and L.E. Doyle. "Cyclostationary signatures for rendezvous in ofdm-based dynamic spectrum access networks". In Proc. IEEE Int. Symposium on New Frontiers in Dynamic Spectrum Access Networks, pp. 220-231. 2001.

[63] P.D. Sutton, J. Lotze, K.E. Nolan and L.E. Doyle. "Cyclostationary signature detection in multipath rayleigh fading environments". In Proc. IEEE Int. Conf. Cognitive Radio Oriented Wireless Networks and Commun. 2007.
[64] M.K. Tsatsanis and G.B. Giannakis. "Transmitter induced cyclostationarity for blind channel equalization". IEEE Trans. Signal Processing. Vol. 45, Issue 7, pp. 785794. 1997.

[65] A. Tkachenko, D. Cabric and R.W. Brodersen. "Cyclostationary feature detector experiments using reconfigurable BEE2”. In Proc. IEEE Int. Symposium on New Frontiers in Dynamic Spectrum Access Networks, pp. 216-219. 2007.

[66] Inc. Std. IEEE 802.11b. "Supplement to ieee standard for information technology telecommunications and information exchange between systems - local and metropolitan area networks - specific requirements part 11: wireless lan medium access control (mac) and physical layer (phy) specifications: high-speed physical layer extension in the $2.4 \mathrm{GHz}$ band". The institute of electrical and electronics engineering. Technical report, IEEE. 1999.

[67] T. Farnham, G. Clemo, R. Haines, E. Seidel, A. Benamar, S. Billington, N. Greco, N. Drew, T. Le, B. Arram and P. Mangold. "Ist-trust: A perspective on the reconfiguration of future mobile terminals using software download". In Proc. IEEE Int. Symposium on Personal, Indoor and Mobile Radio Commun, pp.10541059. 2000.

[68] M. Mehta, N. Drew, G. Vardoulias, N. Greco and C. Niedermeier. "Reconfigurable terminals: an overview of architectural solutions". IEEE Commun. Mag. Vol. 39, Issue 8, pp. 82-89. 2001.

[69] J. Palicot and C. Roland. "A new concept for wireless reconfigurable receivers". IEEE Commun. Mag. Vol. 41, Issue 7, pp. 124-132. 2003.

[70] A.F. Cattoni, I. Minetti, M. Gandetto, R. Niu, P.K. Varshney and C.S. Regazzoni. "A spectrum sensing algorithm based on distributed cognitive models". In Proc. SDR Forum Technical Conference. 2006.

[71] M. Gandetto and C. Regazzoni. "Spectrum sensing: A distributed approach for cognitive terminals". IEEE J. Select. Areas Commun. Vol. 25, Issue 3, pp. 546-557. 2007.

[72] M. Gandetto, M. Guainazzo and C.S. Regazzoni. "Use of time-frequency analysis and neural networks for mode identification in 
a wireless software-defined radio approach". EURASIP Journal on Applied Signal Processing, pp. 1778-1790. 2004.

[73] M. Gandetto, M. Guainazzo, F. Pantisano and C.S. Regazzoni. "A mode identification system for a reconfigurable terminal using wigner distribution and non-parametric classifiers". In Proc. IEEE Global Telecomm. Conf. Vol. 4, pp. 2424-2428. 2004.

[74] M. Oner and F. Jondral. "Cyclostationaritybased methods for the extraction of the channel allocation information in a spectrum pooling system". In Proc. IEEE Radio and Wireless Conf., pp. 279-282. 2004.

[75] Z. Tian and G.B. Giannakis. "A wavelet approach to wideband spectrum sensing for cognitive radios”. In Proc. IEEE Int. Conf. Cognitive Radio Oriented Wireless Networks and Commun. 2006.

[76] Z. Tian and G. Giannakis. "Compressed sensing for wideband cognitive radios". In Proc. IEEE Int. Conf. on Acoustics, Speech, and Signal Processing. Vol. 4, pp. 1357-1360. 2007.
[77] Y. Youn, H. Jeon, J. Choi and H. Lee. "Fast spectrum sensing algorithm for 802.22 wran systems". In Proc. IEEE Int. Symp. Commun. and Information Techn., pp. 960-964. 2006.

[78] Y. Hur, J. Park, K. Kim, J. Lee, K. Lim, C. Lee, H. Kim and J. Laskar. "A cognitive radio (CR) testbed system employing a wideband multiresolution spectrum sensing (MRSS) technique". In Proc. IEEE Veh. Technol. Conf., pp. 1-5. 2006.

[79] K. Challapali, S. Mangold and Z. Zhong. "Spectrum agile radio: Detecting spectrum opportunities". In Proc. Int. Symposium on Advanced Radio Technologies. 2004.

[80] Y.Zeng and Y.-C. Liang. "Covariance based signal detections for cognitive radio". In Proc. IEEE Int. Symposium on New Frontiers in Dynamic Spectrum Access Networks, pp. 202-207. 2007.

[81] B. Wild and K. Ramchandran. "Detecting primary receivers for cognitive radio applications". In Proc. IEEE Int. Symposium on New Frontiers in Dynamic Spectrum Access Networks, pp. 124-130. 2005. 